\title{
PHOTON CORRELATION AND STATISTICS OF SPONTANEOUS PARAMETRIC DOWN-CONVERSION PUMPED BY BLUE LED IN $\mathrm{LiIO}_{3}$ CRYSTAL
}

\author{
J. Galinis ${ }^{\text {a }}$, M. Karpiński ${ }^{\text {b }}$, G. Tamošauskas ${ }^{\text {a }}$, K. Dobek ${ }^{c, d}$, and A. Piskarskas ${ }^{\text {a }}$ \\ a Department of Quantum Electronics, Vilnius University, Sauletekio 9-III, LT-10222 Vilnius, Lithuania \\ ${ }^{\mathrm{b}}$ Faculty of Physics, University of Warsaw, Hoża 69, 00-681 Warsaw, Poland \\ ' Institute of Physics, Nicolaus Copernicus University, Grudziądzka 5/7, 87-100 Toruń, Poland \\ ${ }^{\mathrm{d}}$ Faculty of Physics, Adam Mickiewicz University, Umultowska 85, 61-614 Poznań, Poland \\ E-mail: justinas.galinis@gmail.com
}

Received 6 January 2012; revised 16 April 2012; accepted 22 June 2012

\begin{abstract}
We report on the measurement of photon coincidence and photon number statistics of the spontaneous parametric down-converted radiation excited by incoherent pump. A high ratio of photon coincidences in case of multimode acquisition suggests that the present technique may find application in quantum experiments. Experimental and numerical simulation results confirmed that the coincidence ratio in a photon counting experiment is tightly coupled to the coherence of the pump beam. An experiment on verification of statistical properties of the down-converted radiation allowed us to evaluate the afterpulse generation probability of the photon counters.
\end{abstract}

Keywords: spontaneous parametric down-conversion, biphoton field, photon correlation, photon statistics

PACS: 42.50.Ar, 42.65.Lm

\section{Introduction}

A new branch of optical experiments broke out in the 1960s due to the invention of the laser. During the first decade of the laser era the spontaneous parametric down-conversion (SPDC) was observed both theoretically and experimentally [1], 21. Soon SPDC found its applications in quantum optics as a two-photon source for violation of Bell's inequalities [3], quantum cryptography [4], tomography [5], lithography [6], quantum computing [⿰], and metrology [8]. Entangled photons for quantum optics experiments can be created in biaxial crystals [9] or with electrically driven entangled-lightemitting diodes [10].

The very first experimental observation of SPDC pumped by an incoherent source was performed using a mercury lamp [11] - a spatially incoherent but very narrow frequency bandwidth light source - which imitated laser radiation. Re- cently our group showed that SPDC generation was effective with both spatially and frequency incoherent source. We observed SPDC [12] pumped by the blue light emitting diode (LED) and measured a sufficient level of multimode fibre coupled photons from such a two-photon light source [13]. These results open perspectives for the incoherently pumped two-photon sources to be used in quantum optics experiments.

In this paper we present experimental and numerical results of SPDC photon coincidence probabilities and their dependence on pump spatial spectra, which are the measure of spatial coherence. Photon pairs were generated using the standard methodology [14], based on SPDC in a Type I nonlinear crystal. We found that coincidences were not linearly linked to the spatial bandwidth of the pump beam and recorded the highest value of $11.5 \%$ coincidence to single photon count ratio. 
Using the experimental set-up built for photon coincidence measurements, the statistics of photon number probability distribution can also be measured with only minor modifications. The measurement of the photon number distribution is a commonly used method used to gain complete statistical information about the light source [15. Poissonian distribution is expected for any statistics of the source itself when the response time of the photon counters is significantly longer than the coherence time of the source. The multimode SPDC field photon statistics are described by the Poissonian distribution [16] when pumped with coherent radiation. A possibility to generate SPDC photons pumped by an incoherent source raises the question whether the statistics of the downconverted radiation is affected by the different photon statistics of the pump. The difference may occur due to incoherent light used as a pump source in the present experiment being described by a BoseEinstein photon number distribution.

Statistical information may be used to demonstrate nonclassicality of the light field, such as in photon bunching or antibunching [17]. Almost all statistical experiments were performed using only laser sources, which are known to be Poissonian light sources [18]. Some observers reported photon distribution differences between thermal and Poissonian statistics [19]. Some observations were made using statistically modified laser pulses with resulting sub-Poissonian statistics, [20] but there are no works, to the best of our knowledge, where the photon number statistics from a nonlinear crystal source pumped by true incoherent light has been investigated. We exploited the possibility to record information of the photon statistics of incoherently pumped SPDC even if our experimental capabilities were limited, and we are presenting the results of the research in section 4 of the paper.

\section{Experimental set-up}

The scheme of our experimental set-up is shown in Fig. 1. We used a both spatially and spectrally incoherent blue LED (LZ1-10UA05 from LedEngin, Inc.) with $403.5 \mathrm{~nm}$ central wavelength and $14 \mathrm{~nm}$ FWHM bandwidth as a pump source. Details of the LED pump source as well as of light detection part of the experimental set-up can be found in the recent paper [13] of our team. The Glan prism

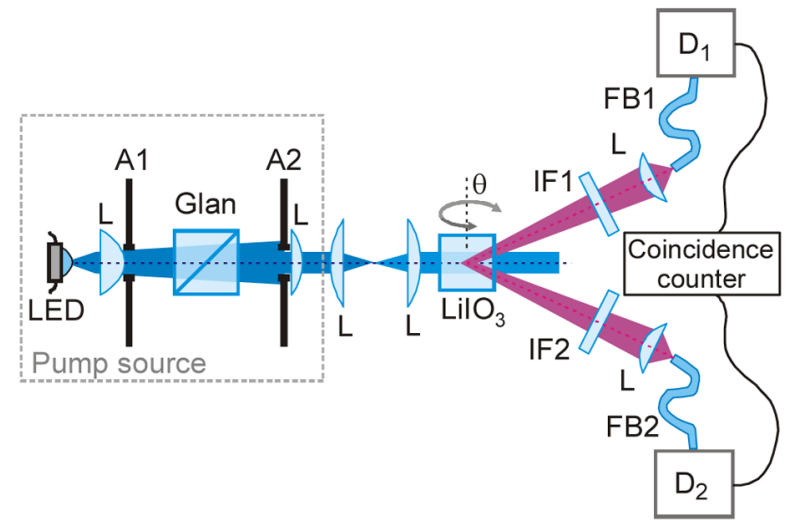

Fig. 1. (Color online) Experimental set-up: $L$ lenses, $A 1$ and $A 2$ apertures, $I F 1$ and $I F 2$ interference filters, $F B 1$ and FB2 fibres, $D_{1}$ and $D_{2}$ photon counters.

was used to linearly polarise the pump ensuring that only the light of extraordinary polarisation is sent to the nonlinear crystal. LED radiation was spatially filtered using a set of hard apertures $A 1$ and $A 2$. Such technique allowed us to independently change the pump beam divergence (aperture $A 1$ ) and the diameter (aperture $A 2$ ), followed by a power change due to the constant radiance of the source. Measurements were performed using four different pump divergences: 9, 15, 25, and 40 mrad.

Biphoton field was generated in a Type $\mathrm{I} \mathrm{LiIO}_{3}$ crystal of $20 \mathrm{~mm}$ length cut at $\theta=35^{\circ}$. The crystal was oriented at $\theta=43.4^{\circ}$ with respect to the pump in order to obtain frequency degeneracy at $800 \mathrm{~nm}$. SPDC field emerged as a $0.1 \mathrm{mrad}$ opening angle cone. Two identical channels for photon collection and registration were mounted symmetrically around the cone axis. Interference filters (IF1 and IF2) transmitted SPDC radiation of $37.5 \mathrm{~nm}$ bandwidth around degeneracy. The biphoton field from these channels was focused by individual lenses into two multimode fibres ( $F B 1$ and $F B 2$ ) of $105 \mu \mathrm{m}$ core diameter and numerical aperture of 0.22 , followed by a pair of PerkinElmer Inc. SPCMAQRH-14-FC photon counters $\left(D_{1}\right.$ and $\left.D_{2}\right)$. Transistor-transistor logic (TTL) pulses from both photon counters were directed to a coincidence circuit (with a coincidence window of $7 \mathrm{~ns}$ ) for photon coincidence measurement.

Photon number statistics in both arms were measured using a $20 \mu$ s detection window for both photon counters separately. Photon counters' signals 
were sent for analysis to the computer configurable electronic unit which delivered the rate of photon coincidences or time stamped record for photon number statistics on demand.

\section{Dependence of the photon coincidence rate on the spatial spectra of the pump}

The first part of the experiment was dedicated to the measurement of photon coincidences. We used a large variety of parameters for the pump beam in order to determine relationships between the properties of the pump and the resulting SPDC radiation: the divergence of the pump was varied from 9 to $40 \mathrm{mrad}$, the pump beam diameter from 0.8 to $8 \mathrm{~mm}$. The power of the pump beam varied accordingly in the range from $1.64 \mu \mathrm{W}$ to $3.26 \mathrm{~mW}$, because of the fixed radiance of the LED whose radiation was transformed into the pump beam by modifying its spatial bandwidth and beam diameter. A single photon rate in a detection channel could be expected to vary proportionally (i. e. by 3 orders of magnitude) if all pump spectral components participated equally in the generation of the SPDC photons and all these photons were created in the cone of spatial width that fitted into the spatial acceptance angle of each detection channel. However, detected single photons varied by less than 2 orders of magnitude only, in the range of $(1-80) \cdot 10^{3}$ photons per second. Such discrepancy between single photon numbers and pump power appears due to specific $\mathrm{LiIO}_{3}$ dispersion characteristics.

First of all, a pump beam can be expressed as a superposition of plane monochromatic waves [12, 13, 21]. Each plane wave, which is a component of the pump, generates its own SPDC cone. Plane waves that are an addition to the existing set of plane waves (when the divergence of the pump is broadened) generate individual parametric cones. The axes of these cones are oriented according to the propagation direction of the appropriate plane wave component. The total SPDC field is an integral of all these separate parametric cones [21], which results in blurring that is dependent on the divergence of the pump [22] or, more exactly, in spreading of the cone axes of individual plane wave components.

Secondly, the refractive index of the extraordinary wave in the $\mathrm{LiIO}_{3}$ crystal strongly depends on the propagation direction in the $\theta$ plane. Therefore, a small change of the plane wave component propagation direction changes the length of the wave vector of this component. This change modifies the angle between the non-collinearly phasematched signal and idler waves, which corresponds to the change of the opening angle of the parametric cone. As a result, the width of the parametric cone is blurred by several factors: the pump wave direction and the opening angle variation due to a broad spatial spectrum of the pump and additionally by the variation of the length of wave-vector of the individual plane wave component of the pump, due to a broad pump wavelength bandwidth. Experimental investigation of the influence of the last factor is out of reach of the used experimental setup; therefore, it will not be discussed in detail.

A parametric waves' ray model is better suited to explain the influence of the pump beam size on the photon count rates. At first glance no dependence on the diameter of the pump beam is expected. The dependences observed in the real experimental setup can be associated with the limited apertures of the lenses collecting light into the fibres. The pump beam can be expressed as a set of rays, similarly to the ray-tracing method. Each ray can act as a pump beam with its individually generated parametric cone. The widening of the pump beam diameter can be understood as introducing additional rays shifted in space away from the central axis; it results in the generation of parametric cones with shifted axes. Changes of the pump beam components' direction associated with spatial spectrum results in variation of the parametric emission cone opening angle, which becomes so different from the mean value that it misses the apertures of the lenses and thus cannot be sent into the fibres. To summarise, both processes that are linked to the pump beam divergence and diameter are nothing else than spatial coherence dependent processes. It is clear that the decreased spatial coherence relatively increases the blurring of the parametric cone, and the number of detected SPDC photons may decrease.

The measured coincidence rate was reliable and high enough for reliable detection: 120-2100 coincidences per second. However, quantum optics experiments require not only a high flux of photon coincidences but also a good quality of a signal or coincidence rate, which can be expressed as a ratio of the numbers of photon coincidences and single 
photons. The main question is how the coincidence rate depends on the spatial properties of the pump field. To answer this question we measured the dependence of the coincidence rate on pump divergence using several different pump beam diameters. The results for three pump beam sizes $(0.8$, 1.8, and $5 \mathrm{~mm}$ diameter) are presented in Fig. 2(a). High coincidence ratio should be achieved when opposite fractions of the parametric cone (with respect to the pump axis) are guided to both photon counters. A wider pump beam causes separate parametric cones, each generated from an individual pump ray, to become so separated in space that they cannot be guided to the photon counters. This leads to the stabilisation of the coincidence rate. Simultaneously, single photons from the cones of different opening angles, resulting from the pump

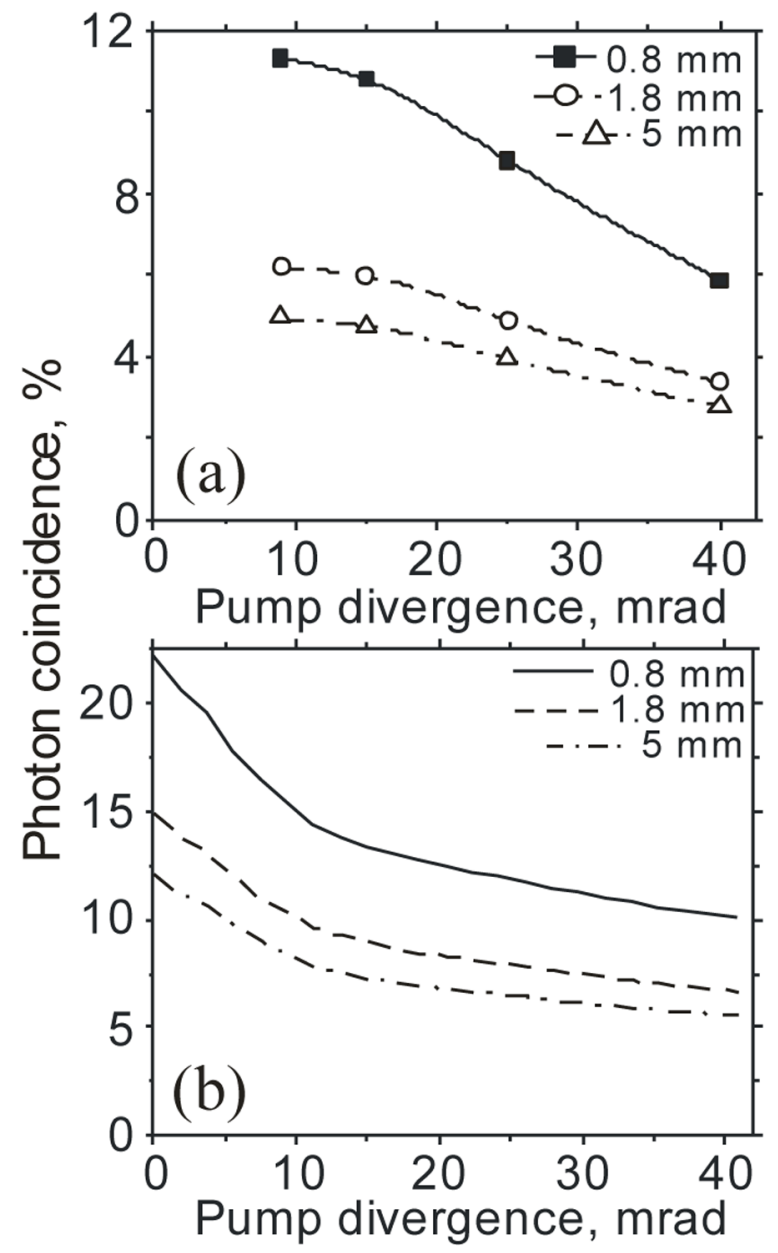

Fig. 2. Dependence of the photon coincidence rate on pump divergence for three pump beam sizes: $0.8 \mathrm{~mm}$ (squares), $1.8 \mathrm{~mm}$ (circles), and $5 \mathrm{~mm}$ (triangles). (a) Experimental results - pumping with LED. (b) Theoretical results - for monochromatic pump beam. wavelength and direction shift, still provide individual photons that can reach the detectors, which decreases the ratio of coincidences to single photon counts. This process is dominant when the beam diameter changes from 0.8 to $1.8 \mathrm{~mm}$. Finally, the relative lateral shift increases so much that there are no more changes in beam diameters above $5 \mathrm{~mm}$, neither in coincidence nor in single photons flux. This leads to the stabilisation of the recorded values of the coincidence to single count ratio. The data recorded with the $8 \mathrm{~mm}$ diameter pump beam is not shown because it coincides almost exactly with the data recorded using the $5 \mathrm{~mm}$ beam.

The photon coincidence rate is also dependent on the pump beam divergence - wider divergence determines a lower coincidence rate. The reason for this dependence is straightforward. A lower pump divergence angle provides a thinner and more symmetric SPDC cone. Thus the generated twin photons deploy more symmetrically around the cone axis. Therefore, the number of detected unpaired photons decreases in comparison with the number of the detected biphotons and as a result the coincidence to single count ratio and the flux become higher. Numerical simulation was performed under conditions similar to experimental but with a monochromatic pump only, in order to compare it with laser radiation. The obtained results, shown in Fig. 2(b), agree well qualitatively with the experimental results acquired with a broadband pump. Differences occur mainly at the small divergence of the pump beam (below $10 \mathrm{mrad}$ ), where the rise of the coincidence ratio in the experiment is limited by the wavelength bandwidth of the pump. The simulation confirms that the diminishing of the coincidence ratio caused by the broadening of the pump divergence is the process of a lower order than the second order process of the pump power boosting, which is determined by the constant radiance of the LED.

Here we can conclude that the divergence of the pump may not be a critical parameter when considering incoherently pumped SPDC. Various visible and ultraviolet LEDs whose optical power can reach a few watts out of a single chip have a frequency bandwidth similar to the one used in the present experiment. The pump power, following the same beam formation strategy, would depend on the second order of the diameter and the divergence of the beam. The divergence can be wide 
enough without causing a significant decrease of the coincidence to single count ratio. As a result, losses of the initial power of the LED would not be high, making the LED an attractive pump source, especially when broad frequency bandwidth or spectral tuning is needed.

Finally, the process efficiency was evaluated when a single-mode fibre was used for collecting the photons. A multimode fibre accepts approx. 4000 spatial modes as estimated from the core diameter and the numerical aperture of the fibre. It is reasonable to expect a significant drop of the single photon flux by the ratio approximately corresponding to the number of spatial modes, when one of the multimode fibres is replaced with a singlemode fibre (Thorlabs $780 \mathrm{HP}$ ) with the $5 \mu \mathrm{m}$ core diameter and numerical aperture of 0.13 . It was assumed that no realignment of the optical systems was necessary while replacing the multimode fibre with the single-mode one because it was expected that the core of the single-mode fibre, whose both diameter and numerical aperture are significantly smaller than those of the multimode fibre, would simply occupy a region somewhere in the middle of the replaced multimode fibre. Measurements were performed with the pump beam of $25 \mathrm{mrad}$ divergence and $3 \mathrm{~mm}$ diameter. Photon flux was $29 \cdot 10^{3}$ singles per second in the multimode fibre and 17.5 singles per second in the single-mode fibre (dark counts were subtracted), obtained from a $600 \mathrm{~s} \mathrm{ac}-$ cumulation time. One can notice that the ratio of photon fluxes in both types of fibres and the estimated number of modes in the multimode fibre are of the same order. This can be explained by simply comparing the core diameters and numerical apertures of the fibres. By comparing the acceptance areas and divergences of the radiation, which can be accepted by the fibre, the ratio of 1270 is obtained, which matches well the experimentally recorded ratio of 1660 . This result shows that the area around the core of the fibre is quite uniformly illuminated.

The recorded coincidence rate is 1.31 coincidences per second with the use of one multi- and one single-mode fibre. The coincidence rate decreased by three orders of magnitude with respect to the configuration when two identical multimode fibres were used (1230 coincidences per second). Comparing the coincidence to single count ratios from both measurements a significant improvement can be seen: the ratio increased from 4.2 to
$7.5 \%$ when referred to the single-mode fibre detection channel. This result matches well with the numerical model design strategy used in our previous paper [13]. The spatial shift of the paired photons with respect to each other is determined by the frequency bandwidth and divergence of the pump. Simulation results of paired photons distribution in the plane of the fibres' tips are shown in Fig. 3. For the sake of simplicity, idealised data presentation is used - it is assumed that the geometry of the non-collinear interaction does not play a role, as it would be the case in a thin crystal. The data shown is obtained as follows: SPDC is generated by the pump and the signal is tracked to see if it fits into the reference fibre and passes the interference filter. The conjugated idler wave is calculated and a point is placed in the second channel fibre's tip plane. The spatial distribution of the idler wave photons is obtained by calculating all frequency and spatial distribution combinations of the pump. The cross-sections of the paired photons' distribution are shown in Fig. $3(\mathrm{a}, \mathrm{b})$ in the $\theta$ and $\varphi$ planes for single-mode (5 $\mu \mathrm{m}$ core diameter)

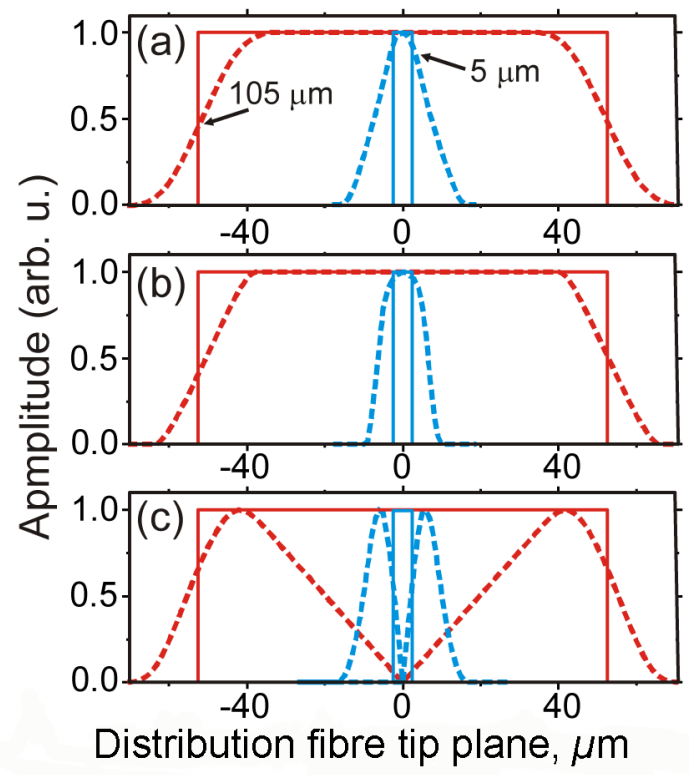

Fig. 3. Density of paired photons in $5 \mu \mathrm{m}$ core diameter single-mode (blue lines online) and $105 \mu \mathrm{m}$ core diameter multimode (red lines online) fibre cross-sections in (a) $\theta$ and (b) $\varphi$ planes, and (c) density in fibre radius. Solid lines correspond to fibre detection area or single photon detection distribution, dashed lines represent conjunct photons distributions. 
and multimode $(105 \mu \mathrm{m})$ fibres, respectively; here $\theta$ and $\varphi$ planes are two orthogonal planes crossing the pump beam. The names of the planes are given in association with angles of crystallographic axes: phase-matching angle lies in the $\theta$ plane. It is clear that for a multimode fibre most of the paired photons fit into the fibre (its detection space is plotted as rectangular). A single-mode fibre detects just a small portion of the conjunct photons - the conjunct photon distribution (Fig. $3(\mathrm{a}, \mathrm{b})$ ) overfills the single-mode fibre borders. The differences between single- and multimode fibres are much more clearly visible in Fig. 3(c) where the paired photon flux density in the radius of the fibre is shown.

Almost all conjunct photons from the singlemode fibre are spread in an area wider than the fibre diameter itself. Nevertheless, this distribution area is smaller than the multimode fibre diameter, which increases the probability for the paired photon to pass into the multimode fibre and thus increases the coincidence probability. Experimental results still differ from the presented simulation (100\% of coincidences referred to a single-mode fibre should be obtained when mixed single-mode and multimode fibres are used). This can be explained by additional blurring in the $\varphi$ plane due to the non-collinear geometry of the experiment. These results clearly indicate that a set-up designed in similar way with the use of two single-mode fibres would not provide a sufficient signal to perform an experiment. Therefore, the use of LEDs as a pump source for SPDC is suitable only for the multimode fibre acceptance experiments.

\section{Measurement of photon number statistics of SPDC pumped by incoherent source}

The measurement of the photon number statistics is one of the tools for the characterisation of light sources [15]. It may provide information on the way radiation is emitted with respect to its coherence and modal content. General solutions for a different type of radiation are well analysed theoretically (see for example [18]), but experimental realisations may be challenging because of the specific requirements for the response speed of the photon counters. It is known that the response time of the detector should be shorter than the light coherence time in order to perform a proper measurement of photon number statistics. Otherwise,
Poissonian statistics will be obtained regardless of the statistics of the optical field [18]. This theoretical prediction is obtained analytically as a general solution independent of any particular experimental realisation.

The detection time interval was set at $20 \mu \mathrm{s}$ in the experiment. The dead time of the counters of 50 ns ensured a sufficient counting capability with less than 1 count in average obtained in the detection time interval. This allowed us to use the avalanche-photodiode-based single photon detectors, which cannot resolve the number of detected photons. However, this time was much longer than the coherence time of the SPDC radiation (60 fs as calculated from the frequency bandwidth predetermined by the interference filter). It was clear that shortening the time interval window should not affect the results of the measurements, because there were no technical possibilities of shortening the response time of the detector down to the value similar to the coherence time of the radiation. Therefore, the statistical distributions obtained in the present experiment must refer not to the source but to the detector statistics, because the detection window is significantly longer than the coherence time of radiation [18]. Statistical distribution of multimode radiation is known to be undistinguishable from Poissonian and even more, statistical properties of SPDC excited by the laser are known to be Poissonian [16]. Taking these conditions into account, the results of the present measurements should not be anything else than Poissonian distribution. Therefore, we decided to perform the photon number statistics measurement to test the consistency of our photon counting measurement apparatus. Additionally, to our best knowledge, no data from the measurements of SPDC photon number statistics excited by an incoherent source has been reported, thus any new results may be useful for the discussion of the influence of the excitation source on the statistical properties of SPDC. Despite the fact that it was anticipated that our experiment would not yield anything else than Poissonian distribution, we exploited the possibility to record photon number statistics experimentally and tested the predictions of the quantum statistics theory in our particular experimental arrangement.

The photon number statistics was measured experimentally for three different divergences of the pump beam: 9,15 , and $40 \mathrm{mrad}$, with the diameter 
of the pump beam fixed at the value of $0.8 \mathrm{~mm}$. The data was recorded as sets of numbers of photon counts in a fixed time window. Two channels were recorded independently because they were already mounted in the experiment and the data was analysed independently for each channel. Histograms for the detection channel $D_{1}$ are shown in Fig. 4 as an example of photon number distributions. The analysis of the data was performed using the built-in functions of the Matlab scientific calculation software package. At first, the hypothesis that the data is compliant with the expected Poissonian distribution was tested using the Pearson's chisquared test. A negative result was obtained, which was unexpected, taking into account the reasoning mentioned at the beginning of this section.

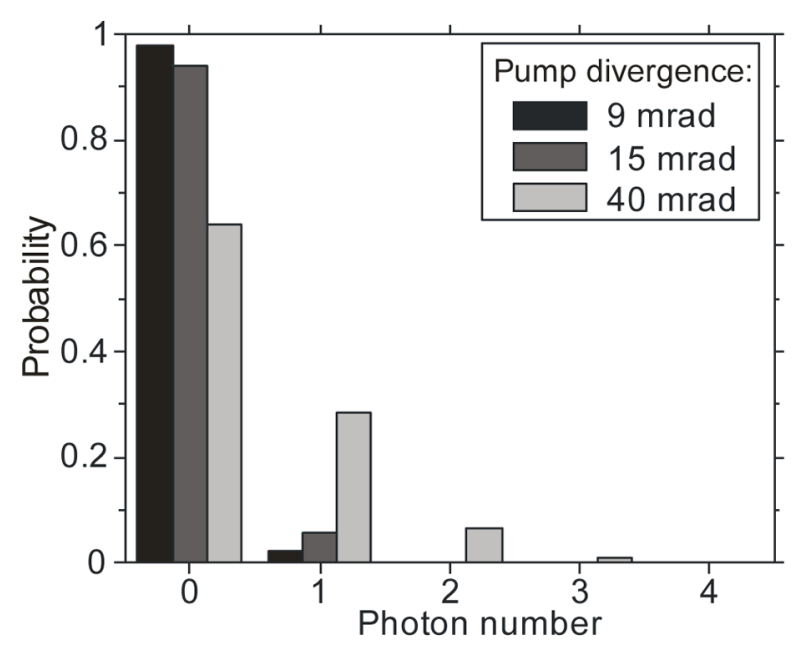

Fig. 4. Photon number distribution for multimode SPDC field in $D_{1}$ channel pumped with incoherent source of 9,15 , and $40 \mathrm{mrad}$ divergence.

As a next step, we tested the data using a negative binomial distribution, motivated by the fact that the pump radiation stems from a thermal source, whose statistics is described particularly by this distribution. Negative binomial distribution can be expressed as

$$
f(k)=\frac{\Gamma(k+r)}{\Gamma(k+1) \Gamma(r)}(1-p)^{r} p^{k} .
$$

Distribution is characterised by just two parameters: number of failures until the experiment is stopped $r$, and success probability in each experi- ment $p$; here $\Gamma(x)$ denotes the gamma function. These two parameters are not directly linked to the properties of the light source. The distribution mode parameter can be introduced for this reason. First of all, secondary parameter $\delta$ describes the average number of counts per "degree of freedom" or per "mode" of the incidence light [18, 23], which is also known as degeneracy degree $\delta=p /(1-p)$. With the use of this parameter we can calculate the distribution mode parameter of the source, taking into account that it is expected to get not an average counts number (with probability equalling $p$ ) but one count (probability 1 ) in each interval:

$$
N_{\text {modes }}=\frac{\delta}{p}=\frac{1}{1-p} \text {. }
$$

With these two parameters $r$ and $N_{\text {modes }}$ it would be possible to characterise the thermal multimode source and make some conclusions in respect of the relations to the spatial spectra of the pump, which is proportional to the number of spatial modes of the pump.

Two negative binomial distribution parameters $r$ and $p$ were calculated and a distribution mode parameter $\left(N_{\text {modes }}\right)$ was estimated. The results are listed in Table 1 for both detection channels $D_{1}$ and $\mathrm{D}_{2}$ for different divergence of the pump beam. The parametric field distribution mode parameter increases together with the divergence of the pump beam. This may look reasonable if we assume that a larger number of spatial modes of the pump participate in a three-wave interaction increasing the total number of parametric field modes.

The estimated distribution mode parameter for all cases varies from 32 to 250 , giving us an answer that statistics of the light field under investigation

Table 1. Statistical parameters of the biphoton field: $r$ and $p$ are binomial distribution parameters (see the text above for details), $N_{\text {modes }}$ is the distribution mode parameter.

\begin{tabular}{c|c|c|c}
\hline $\begin{array}{c}\text { Channel No. and } \\
\text { pump divergence }\end{array}$ & $r$ & $p$ & $N_{\text {modes }}$ \\
\hline$D_{1} 9 \mathrm{mrad}$ & 4.54 & 0.9949 & 196 \\
\hline$D_{1} 15 \mathrm{mrad}$ & 11.2 & 0.9945 & 181 \\
\hline$D_{1} 40 \mathrm{mrad}$ & 111 & 0.996 & 250 \\
\hline$D_{2} 9 \mathrm{mrad}$ & 0.77 & 0.968 & 31.6 \\
\hline$D_{2} 15 \mathrm{mrad}$ & 2.41 & 0.972 & 35.8 \\
\hline$D_{2} 40 \mathrm{mrad}$ & 18.5 & 0.973 & 37 \\
\hline
\end{tabular}


differs a lot from the Poissonian. Obtained results should be explained from a different perspective. First of all, the aggregate distribution of a few Poissonian sources is always Poissonian. We mentioned previously that the laser (the laser has fewer spatial modes compared to the LED) pumped SPDC delivers Poissonian distribution [16]. Therefore, the sum of independently generated same distributions when each may be attributed to a single spatial mode of the pump must also be of the same Poissonian distribution. Secondly, we were examining only spatial modes, but longitudinal or frequency modes should also be taken into account with a simple conclusion that obtained values are most probably wrong. Finally, two identical channels show very different values even if they are pointed to the same source.

The experimental set-up was inspected for the source of deviations that could lead to the disturbed statistical measurement from the perspective of the recently made conclusions. It was shortly perceived that the photon counters themselves may be the source of the problem, since both optical set-ups were identical and the deviation can most probably be attributed to afterpulse generation of the photon counter. Such a problem was analysed in several works related to the detection and correction of afterpulse influence on a measured statistical distribution profile [24, 25]. The idea was, assuming the statistics is Poissonian, to recover statistical distribution by counting empty intervals $n_{0}=0$ and nonempty intervals $n_{\mathrm{e}}>0$ independently, depending on the number of counts in each. The ratio of these two quantities shows distribution related properties: $\frac{n_{0}}{n_{e}}=\frac{P(0)}{\sum P(i)}$, which allows one to find only one unknown parameter - the average number of events in the interval. Therefore, the original Poissonian distribution can be recovered and afterpulse probability retrieved. We decided to exploit an accidentally observed feature - a well detectable impact of afterpulse generation on the parameters of the negative binomial distribution - to calculate the detectors' afterpulse probability.

Numerical simulation was performed in order to confirm whether afterpulse generation probability of the value stated in the datasheet of the product might influence the results similarly to the experimentally obtained effects. The algorithm of the simulation was following. First of all, a data set of Poissonian distributions of similar length and average count rate as obtained in the experiment was numerically generated. The data were modified by adding an extra count to each existing count randomly following the afterpulse generation probability. The negative binomial distribution was fitted to the modified data set. The values of the corresponding distribution mode parameter when afterpulse generation probability varies are shown in Fig. 5 .

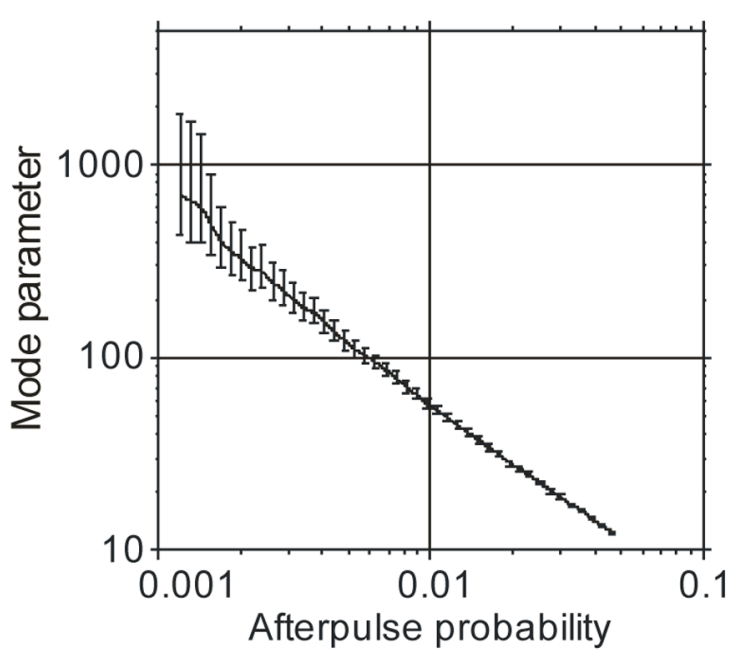

Fig. 5. Distribution mode parameter dependence on afterpulse probability.

This result can explain well our experimental findings. The calculated afterpulse generation probability was $0.31 \%$ for $D_{1}$ and $1.9 \%$ for $D_{2}$, and these values match the ones supplied by the device manufacturer.

Here we can conclude that the experimental arrangement was of insufficient time resolution to record the thermal photon statistics of the source. On the other hand, the distribution mode parameter strongly depends on the counters' afterpulse generation probability. This feature may be used to measure the afterpulse generation probability of the photon counter. The values of mode parameters associated with certain afterpulse probabilities are not constant and increase with the average number of counts in a time interval. Therefore, the suggested method is not universal and different curves should be obtained for different average count 
rates in a time interval. This is not a problem taking into account the computation capabilities of modern computers.

Finally, we can suggest a method to modify the experiment in order to retrieve photon-detector statistics avoiding the impact of photon counters' afterpulse generation. Using photon counters with negligible afterpulse generation can of course solve the problem. An alternative and, from the practical point of view, a more realistic solution can be to use pulsed light instead of continuous wave together with additional gating for the counting electronics. LED can be driven by pulsed power supply in such a way that the light pulse "on" time would be no longer than the dead time of the photon counter and the "off" time longer than the afterpulse generation time delay. The afterpulse generation probability for PerkinElmer Inc. SPCM-AQR series photon counters drops below 0.0005 after $500 \mathrm{~ns}$ delay, which is two orders of magnitude less than the initial value. The dead time of the counter is $50 \mathrm{~ns}$. The suggested LED timing would be $50 \mathrm{~ns}$ switched on and $500 \mathrm{~ns}$ switched off. The impact of afterpulse generation could be excluded this way because these pulses would appear out of the gated time window. The impact of photon counter's dark counts is contracted at the same time because the "off" time would be longer than the gated time which is equivalent to the "on" time. It can be expected that with this modification at least the expected Poissonian statistics could be recorded correctly.

\section{Conclusions}

The biphoton field generation in Type $\mathrm{I} \mathrm{LiO}_{3}$ pumped by an incoherent light source LED was studied experimentally. We observed that the photon coincidence ratio using an incoherent light source is high enough for quantum optics experiments. The coincidence rate depends strongly on pump beam coherence. We showed that the coincidence rate diminishes proportionally to the spatial bandwidth and the size of the pump beam. The maximal coincidence value at the same time is limited by the frequency bandwidth. Therefore, optimal conditions are achievable for a quite broad spatial bandwidth or divergence of the beam in case of an incoherent pump.
Statistical analysis of the photon counts retrieved that the experiment was strongly affected by the afterpulse generation of the photon counters. The number of modes obtained from fitting a negative binomial distribution may be used to determine the afterpulse generation probability of the photon counter when the used source is of Poissonian distribution.

\section{Acknowledegments}

This work was supported by the Foundation for Polish Science TEAM Project and the Polish Ministry of Science grant No. N N202 482439. The experimental part was carried out at the National Laboratory for Atomic, Molecular and Optical Physics in Toruń.

\section{References}

[1] W.H. Louisell, A. Yariv, and A.E. Siegman, Quantum fluctuations and noise in parametric processes, Phys. Rev. 124, 1646-1654 (1961).

[2] S.E. Harris, M.K. Oshman, and R.L. Byer, Observation of tunable parametric fluorescence, Phys. Rev. Lett. 18, 732-734 (1967).

[3] F. Sciarrino, G. Vallone, G. Milani, A. Avella, J. Galinis, R. Machulka, A.M. Perego, K.Y. Spasibko, A. Allevi, M. Bondani, and P. Mataloni, High degree of entanglement and nonlocality of a twophoton state generated at $532 \mathrm{~nm}$, Eur. Phys. J. Spec. Top. 199, 111-125 (2011).

[4] N. Gisin, G. Ribordy, W. Tittel, and H. Zbinden, Quantum cryptography, Rev. Mod. Phys. 74, 145195 (2002).

[5] S. Cialdi, F. Castelli, and M.G.A. Paris, Properties of entangled photon pairs generated by a CW laser with small coherence time: theory and experiment, J. Mod. Opt. 56, 215 (2009).

[6] Q. Sun, P.R. Hemmer, and M.S. Zubairy, Quantum lithography with classical light: generation of arbitrary patterns, Phys. Rev. A 75, 065803 (2007).

[7] T. Sleator and H. Weinfurter, Realizable universal quantum logic gates, Phys. Rev. Lett. 74, 40874090 (1995).

[8] Z.Zhao, K.A. Meyer, W.B. Whitten, and R.W. Shaw, Optical absorption measurements with parametric down-converted photons, Anal. Chem. 80, 7635 (2008).

[9] A. Halevy, E. Megidish, L. Dovrat, H.S. Eisenberg, P. Becker, and L. Bohaty, The biaxial nonlinear crystal $\mathrm{BiB}_{3} \mathrm{O}_{6}$ as a polarization entangled photon source using non-collinear type-II parametric down-conversion, Opt. Express 19, 20420-20434 (2011). 
[10] C.L. Salter, R.M. Stevenson, I. Farrer, C.A. Nicoll, D.A. Ritchie, and A.J. Shields, An entangled-lightemitting diode, Nature 465, 594-597 (2010).

[11]D.L. Weinberg, Observation of optical parametric noise pumped by a mercury lamp, J. Appl. Phys. 41, 4239-4240 (1970).

[12]G. Tamošauskas, J. Galinis, A. Dubietis, and A. Piskarskas, Observation of spontaneous parametric down-conversion excited by high brightness blue LED, Opt. Express 18, 4310-4315 (2010).

[13]J. Galinis, M. Karpiński, G. Tamošauskas, K. Dobek, and A. Piskarskas, Photon coincidences in spontaneous parametric down-converted radiation excited by a blue LED in bulk $\mathrm{LiIO}_{3}$ crystal, Opt. Express 19, 10351-10358 (2011).

[14] C.K. Hong, Z.Y. Ou, and L. Mandel, Measurement of subpicosecond time intervals between two photons by interference, Phys. Rev. Lett. 59, 20442046 (1987).

[15]F.T. Arecchi, Measurement of the statistical distribution of Gaussian and laser sources, Phys. Rev. Lett. 15, 912-916 (1965).

[16] M. Avenhaus, H.B. Coldenstrodt-Ronge, K. Laiho, W. Mauerer, I.A. Walmsley, and C. Silberhorn, Photon number statistics of multimode parametric down-conversion, Phys. Rev. Lett. 101, 053601 (2008).

[17]E. Waks, B.C. Sanders, E. Diamanti, and Y. Yamamoto, Highly nonclassical photon statistics in parametric down-conversion, Phys. Rev. A 73, 033814 (2006).
[18]J.W. Goodman, Statistical Optics (John Willey \& Sons, Inc., New York, 2000) pp. 465-490.

[19] L. Dovrat, M. Bakstein, D. Istrati, A. Shaham, and H.S. Eisenberg, Measurements of the dependence of the photon-number distribution on the number of modes in parametric down-conversion, Opt. Express 20, 2266-2276 (2012).

[20] M. Curty, T. Moroder, X. Ma, and N. Lütkenhaus, Non-Poissonian statistics from Poissonian light sources with application to passive decoy state quantum key distribution, Opt. Lett. 34, 32383240 (2009).

[21]J. Galinis, G. Tamošauskas, and A. Piskarskas, Modeling of photon coincidence and dispersive properties of spontaneous parametric down-converted field excited by incoherent source, Opt. Commun. 285, 1289 (2011); http://dx.doi.org/10.1016/j.optcom.2011.10.072.

[22]H. Di Lorenzo Pires, F.M.G.J. Coppens, and M.P. van Exter, Type-I spontaneous parametric down-conversion with a strongly focused pump, Phys Rev. A 83, 033837 (2011).

[23] L. Mandel and E. Wolf, Optical Coherence and Quantum Optics (Cambridge University Press, New York, 1995).

[24] L. Campbell, Afterpulse measurement and correction, Rev. Sci. Instrum. 63, 5794 (1992).

[25] G.F. Zhang, S.L. Dong, T. Huang, Y. Liu, J. Wang, L.T. Xiao, and S.T. Jia, Photon statistical measurement of afterpulse probability, Int. J. Mod. Phys. B 22, 1840-1845 (2008).

\title{
PARAMETRINĖS FLUORESCENCIJOS, ŽADINAMOS MĖLYNU ŠVIESOS DIODU LiIO $_{3}$ KRISTALE, FOTONŲ KORELIACIJA IR STATISTIKA
}

\author{
J. Galinis ${ }^{\text {a }}$, M. Karpiński ${ }^{b}$, G. Tamošauskas ${ }^{\text {a }}$, K. Dobek ${ }^{\text {c,d }}$, A. Piskarskas ${ }^{\text {a }}$ \\ ${ }^{a}$ Vilniaus universiteto Kvantines elektronikos katedra, Vilnius, Lietuva \\ ${ }^{\mathrm{b}}$ Varšuvos universiteto Fizikos fakultetas, Varšuva, Lenkija \\ 'Mikalojaus Koperniko universiteto Fizikos institutas, Torūné, Lenkija \\ ${ }^{d}$ Adomo Mickevičiaus universiteto Fizikos fakultetas, Poznanè, Lenkija
}

\section{Santrauka}

Darbe pateikiami parametrinès fluorescencijos, žadinamos nekoherentiniu kaupinimu, fotonų koreliacijos ir statistikos matavimų rezultatai. Didelis sutampančių fotonų srautas daugiamodžio šviesolaidžio detekcijos atveju parodo, kad pateiktas bifotonio lauko generavimo metodas gali büti taikomas kvantiniuose eksperi- mentuose. Eksperimentiniai ir skaitmeninio modeliavimo rezultatai patvirtina, kad fotonų sutapimo tikimybè glaudžiai siejasi su kaupinimo pluošto koherentiškumu. Parametrinès fluorescencijos fotonų statistikos rezultatai leido ịvertinti naudotų fotonų detektorių postimpulsų tikimybę. 